\title{
sciendo
}

\section{FTO AND IRX3 GENES ARE NOT PROMISING MARKERS FOR OBESITY IN LABRADOR RETRIEVER DOGS*}

\author{
Adrian Grzemski ${ }^{1}$, Monika Stachowiak ${ }^{1}$, Krzysztof Flisikowski², Monika Mankowska', Paulina \\ Krzeminska ${ }^{1}$, Maciej Gogulski ${ }^{3}$, Roman Aleksiewicz ${ }^{4}$, Maciej Szydlowski ${ }^{1}$, Marek Switonski ${ }^{1}$, Joanna \\ Nowacka-Woszuk ${ }^{1 *}$ \\ ${ }^{1}$ Department of Genetics and Animal Breeding, Poznan University of Life Sciences, Wołyńska 33, \\ 60-637 Poznań, Poland \\ ${ }^{2}$ Chair of Livestock Biotechnology, Technische Universität München, Liesel-Beckmannstr. 1, \\ 85354 Freising, Germany \\ ${ }^{3}$ University Centre for Veterinary Medicine, Poznan University of Life Sciences, Szydłowska 43, \\ 60-656 Poznań, Poland \\ ${ }^{4}$ Department of Internal Medicine and Veterinary Diagnostics, Poznan University of Life Sciences, \\ Wołyńska 35, 60-637 Poznań, Poland \\ •Corresponding author: joanna.nowacka-woszuk@up.poznan.pl
}

\begin{abstract}
Obesity is a serious problem in numerous dog breeds, but knowledge of its hereditary background is scarce. On the contrary, numerous DNA polymorphisms associated with human obesity have been identified, with the strongest effect being demonstrated for $F T O$ gene. We used targeted nextgeneration sequencing (tNGS) to search for polymorphisms in the region harboring FTO and IRX3 in 32 Labrador dogs. Moreover, we investigated the selected regions of FTO and IRX3, orthologous to the human regions associated with obesity, in 165 Labradors. For all dogs, the following information was available: age, sex, gonadal status, body weight, and body conformation score (BCS). The use of tNGS revealed 12,217 polymorphisms, but none of these obtained significance when lean and obese dogs were compared. Study of two SNPs in the 5'-flanking region of FTO in 165 dogs - creating two upstream reading frames (uORFs) - also showed no association with body weight and BCS but suggested the need for improvement in FTO annotation. No polymorphism was found in the 5'UTR of IRX3. Additionally, no differences of CpG islands methylation status between lean and obese dogs were found. Our study suggests that FTO and IRX3 are not useful markers of obesity in Labrador dogs.
\end{abstract}

Key words: dog, obesity, DNA methylation, FTO, IRX3

Obesity is a serious health problem in dogs and is mainly a consequence of diet, limited physical activity, age, sex, and neutering (Gossellin et al., 2007; German,

*This work was supported by the National Science Centre (Poland): projects no. 2013/09/B/ NZ2/02208 and 2016/23/B/NZ2/01762. 
2016). Some breeds (e.g., Labrador retrievers, Beagles, and Cocker spaniels) are especially predisposed to this condition, suggesting the presence of genetic or epigenetic variants responsible for excessive adipose accumulation.

The vast majority of canine hereditary diseases, particularly monogenic disorders, have clinical and molecular analogues in humans (Switonski, 2014; van Steenbeek et al., 2016). Among polygenic disorders, canine obesity is considered an important model for its human counterpart (Osto and Lutz, 2015; Stachowiak et al., 2016; Yeo, 2017), although knowledge of the genetic background of obesity in dogs remains limited. Markers associated with canine predisposition to obesity can be identified in several ways (Switonski and Mankowska, 2013), but to date only the candidate gene approach, based on human obesity markers (Pigeyre et al., 2016), has been used. These studies revealed that the polymorphisms of POMC (Raffan et al., 2016; Mankowska et al., 2017 a) and TNF (Mankowska et al., 2016) are associated with canine obesity, whereas other strong candidates, such as MC4R (Mankowska et al., 2017 b) are not.

Among the genes associated with human obesity, the FTO encoding fat mass and obesity-associated protein, has the strongest effect (Frayling et al., 2007). The genome-wide association studies (GWAS) performed in different human populations have confirmed FTO's association with body mass index (BMI) (Loos and Yeo, 2014). It has been shown that the SNP in the first intron of FTO occurs within the enhancer for the iroquois homeobox 3 (IRX3) gene, which plays a role in adipocyte differentiation (Smemo et al., 2014). Both genes are located near to each other on canine chromosome 2 (CFA2).

DNA methylation of some markers is associated with a risk of obesity in humans (Huang et al., 2015). For example, in obese children, the FYN gene is hypermethylated while PIWIL4 and TAOK3 are hypomethylated. Usefully, the DNA methylation pattern in blood provides a good representation of the methylation profile in adipocytes (Crujeiras et al., 2017) and this allows conducting such analysis in an non-invasive manner.

In this study, we searched for DNA variants and differentially methylated $\mathrm{CpG}$ islands in a positional candidate region of CFA2, which overlaps FTO and IRX3, and which could be associated with body weight and body condition score (BCS) in Labrador dogs.

\section{Material and methods}

\section{Animals}

Three sample sets of Labrador dogs were used: A $(\mathrm{N}=32), \mathrm{B}(\mathrm{N}=165)$, and $\mathrm{C}$ $(\mathrm{N}=28$, Table 1). The condition of the animals was described according to the commonly used 5-score BCS system, where BCS3 represents normal weight, and BCS4 and BCS5 represent overweight and obesity, respectively (https:/www.aaha. org/public_documents/professional/guidelines/weightmgmt_bodyconditionscoring. pdf). Animals of both sexes were included and age (minimum 12 months), weight 
(only dogs with 25-50 kg of body weight), and gonadal status (neutered or intact) were recorded. Distribution of the dogs with different BCS, according to age and weight, is shown in Figure 1. Blood samples were exclusively collected from dogs without obesity-related diseases which could affect body weight. Genomic DNA was isolated from whole blood samples using the MasterPure DNA Purification Kit for Blood Version II (Epicentre, Illumina), and quality and quantity were checked on a Nanodrop 2000 (ThermoFisher Scientific). Samples were collected according to standard Polish veterinary protocols in veterinary clinics during routine visits, with the consent of the owners and the approval of the local Bioethical Commission for Animal Care and Use in Poznan, Poland (31/2013). No pedigree information was available.

Table 1. Structure of the three sample sets of Labrador dogs

\begin{tabular}{|c|c|c|}
\hline Sample set & Structure & Type of analysis \\
\hline $\mathrm{AN}=32$ & $\begin{array}{l}16 \text { healthy }(\mathrm{BCS}=3) ; 8 \text { females, } 4 \text { neutered individuals; Age: } \\
\text { mean } 39 \text { months, median } 30 \text { months, range } 12-96 \text { months; } \\
\text { Body weight: mean } 31 \mathrm{~kg} \text {, median } 30.5 \mathrm{~kg} \text {, range } 26-40 \mathrm{~kg} \\
16 \text { obese dogs }(\mathrm{BCS}=5) ; 9 \text { females, } 2 \text { neutered individu- } \\
\text { als; Age: mean } 69 \text { months, median } 71 \text { months, range } \\
24-108 \text { months; Body weight: mean } 43 \mathrm{~kg} \text {, median } 43 \mathrm{~kg} \text {, } \\
\text { range } 38-50 \mathrm{~kg}\end{array}$ & Targeted NGS \\
\hline \multirow[t]{3}{*}{$\mathrm{B} N=165$} & $\begin{array}{l}44 \text { healthy dogs }(\mathrm{BCS}=3) ; 26 \text { females, } 9 \text { individuals neu- } \\
\text { tered; Age: mean } 44 \text { months, median } 30 \text { months, range } \\
12-171 \text { months; Body weight: mean } 31 \mathrm{~kg} \text {, median } 30 \mathrm{~kg} \text {, } \\
\text { range } 22-40 \mathrm{~kg}\end{array}$ & $\begin{array}{l}\text { DNA } \\
\text { Sanger sequencing }\end{array}$ \\
\hline & $\begin{array}{l}80 \text { overweight dogs }(\mathrm{BCS}=4) ; 42 \text { females, } 14 \text { neutered; Age: } \\
\text { mean } 61 \text { months, median } 48 \text { months, range } 12-144 \text { months; } \\
\text { Body weight: mean } 36 \mathrm{~kg} \text {, median } 36 \mathrm{~kg} \text {, range } 30-46 \mathrm{~kg}\end{array}$ & \\
\hline & $\begin{array}{l}41 \text { obese dogs }(\mathrm{BCS}=5) ; 23 \text { females, } 9 \text { neutered indi- } \\
\text { viduals; Age: mean } 79 \text { months, median } 79 \text { months, range } \\
24-180 \text { months; Body weight: mean } 41 \mathrm{~kg} \text {, median } 40 \mathrm{~kg} \text {, } \\
\text { range } 35-46 \mathrm{~kg}\end{array}$ & \\
\hline \multirow[t]{2}{*}{$\mathrm{C} \mathrm{N}=28$} & $\begin{array}{l}15 \text { healthy dogs }(\mathrm{BCS}=3) ; 7 \text { females, } 4 \text { individuals neutered; } \\
\text { Age: mean } 51 \text { months, median } 48 \text { months, range } 12-96 \text { months; } \\
\text { Body weight: mean } 33 \mathrm{~kg} \text {, median } 33 \mathrm{~kg} \text {, range } 27-40 \mathrm{~kg}\end{array}$ & $\begin{array}{l}\text { DNA methylation } \\
\text { analysis } \\
\text { by pyrosequencing }\end{array}$ \\
\hline & $\begin{array}{l}13 \text { obese dogs }(\mathrm{BCS}=5) ; 9 \text { females, } 1 \text { neutered individual; Age: } \\
\text { mean } 60 \text { months, median } 60 \text { months, range } 24-108 \text { months; } \\
\text { Body weight: mean } 43 \mathrm{~kg} \text {, median } 44 \mathrm{~kg} \text {, range } 35-50 \mathrm{~kg}\end{array}$ & \\
\hline
\end{tabular}

\section{Targeted next-generation sequencing (tNGS)}

Targeted resequencing was applied using a $2.1 \mathrm{M}$ custom-designed sequence capture array (NimbleGen, Roche). DNA samples $(1 \mu \mathrm{g})$ were sonicated using a Bioruptor (Diagenode) to obtain fragments of the recommended lengths (200$800 \mathrm{bp}$ ). The end-repair, A-tailing and adapter ligation steps were performed using 
a KAPA Library Preparation Kit (KAPA Biosystems), following the manufacturer's recommendations. Three samples were multiplexed in each library. The SeqCap EZ Library kit (NimbleGene, Roche) was used to hybridize the capture probes with selected genomic region following separation using a SeqCap EZ Pure Capture Bead Kit (Roche). The libraries were then enriched with an LM-PCR Master Mix (Roche). Size distribution and library quality were verified using the Agilent DNA 1000 kit on the 2100 Bioanalyzer (Agilent). The concentration of the final library was determined using the Qubit 2.0 fluorometer (ThermoFisher Scientific) and normalized to 10 pmol. PhiX genome DNA was used as an internal control (5\% concentration of the final library). Sequencing runs were performed on the MiSeq platform (Illumina) using the MiSeq Reagent Kit version 2, 2×250 bp (Illumina), following the manufacturer's protocol. Reads were trimmed with Trimmomatic software (Bolger et al., 2014) and aligned to the reference dog genome (CanFam3.1) using BWA software (Li and Durbin, 2010). Postalignment processing (fixing paired read alignment, removal of duplicates, and collection of alignment metrics) was performed with Picard (https://broadinstitute.github.io/picard/). GATK software was used to call small variants (SNPs, indels) (Van der Auwera et al., 2013). We rejected variants with less than twelve informative reads and low GATK quality parameters.

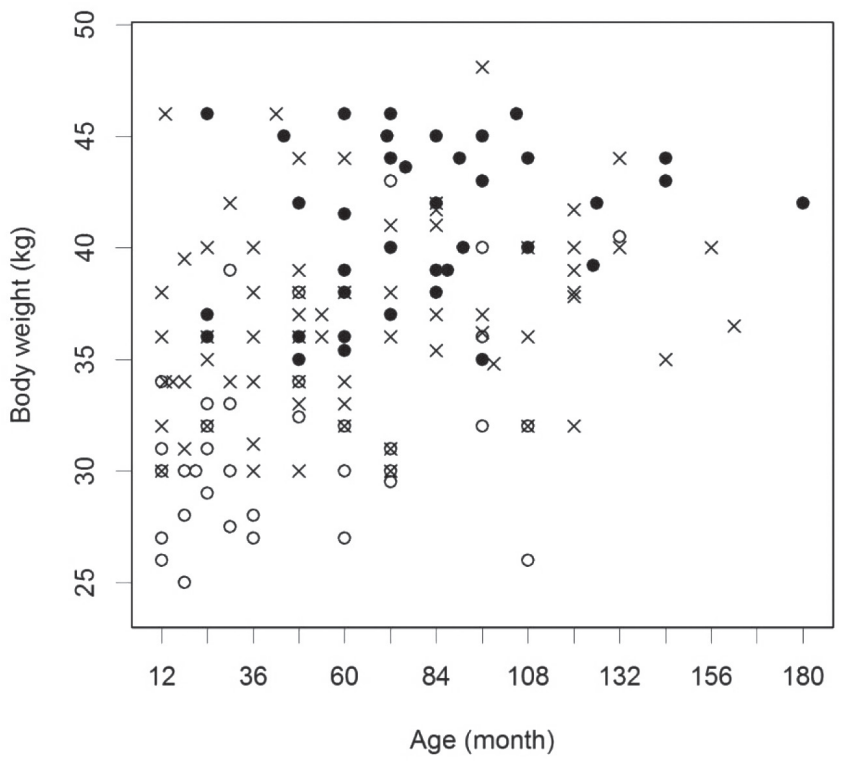

Figure 1. Relation between age, body weight, and body condition score (BCS) in 165 Labrador dogs. Circles: lean dogs $(\mathrm{BCS}=3)$; crosses: overweight dogs $(\mathrm{BCS}=4)$; filled circles: obese dogs $(\mathrm{BCS}=5)$ 

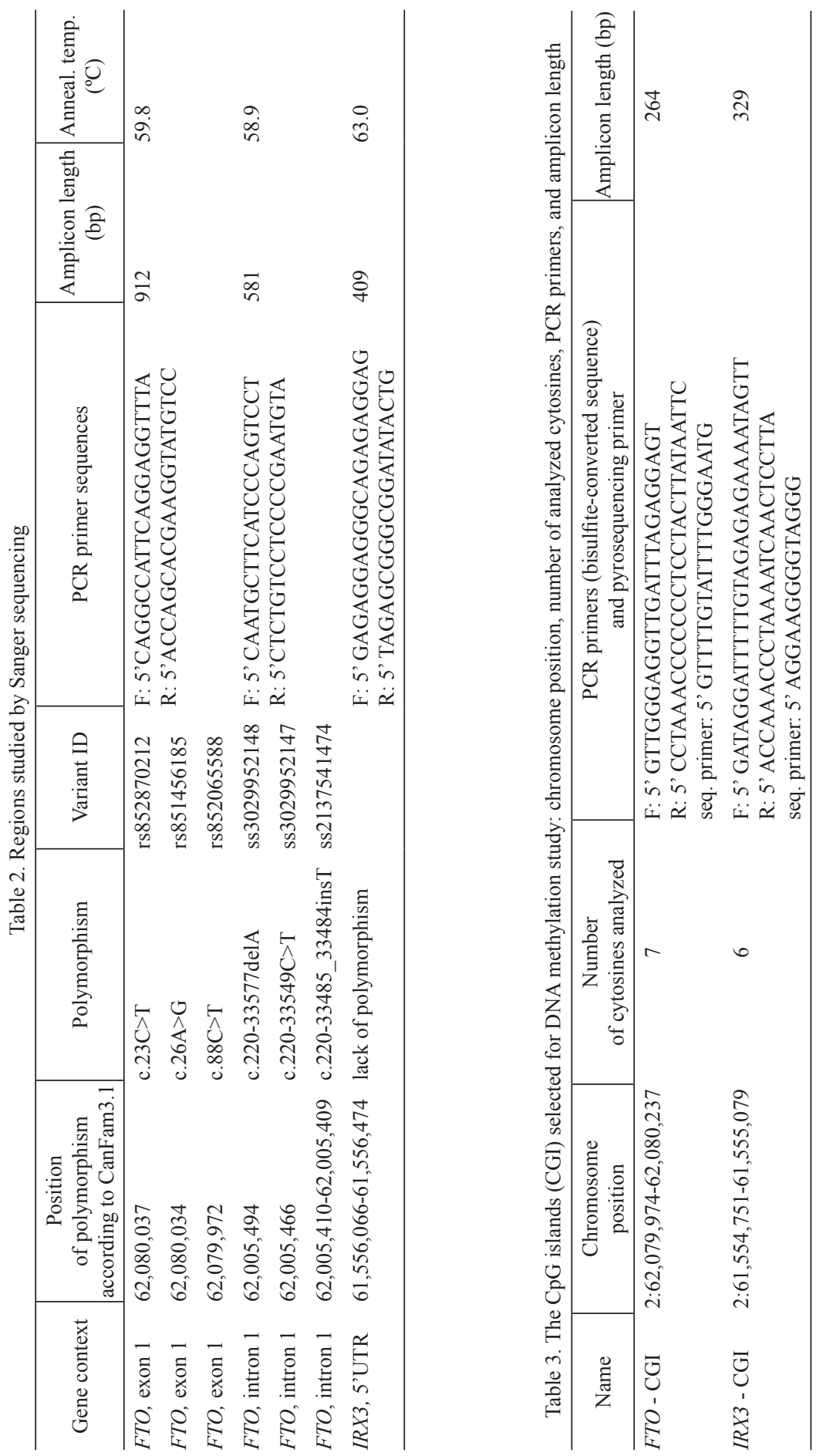


\section{Sanger DNA sequencing}

The PCR amplification primers, amplicon lengths, and annealing temperatures, used in detecting the polymorphisms, are shown in Table 2. The amplicons were purified using alkaline phosphatase and exonuclease I (ThermoFisher Scientific). Amplification was then performed using a BigDye Terminator v3.1 Cycle Sequencing Kit (Life Technologies). Finally, after purification on a Sephadex G-50 (Sigma), capillary electrophoresis was run on a Genetic Analyzer 3130 (Applied Biosystems). Sequences were analyzed using DNAStar software (Lasergene).

\section{In silico studies}

We performed in silico analysis of the genomic regions overlapping the identified polymorphisms in order to search for possible functional relevance. We used BLAST software to compare the canine (CanFam3.1) genomic sequence with the human one (GRCh38) (Zhang et al., 2000). RepeatMasker software (http://repeatmasker. org) was used for repetitive element masking. MatInspector software (Genomatix) was used to search for potential transcription factor binding sites. StarORF software (http://star.mit.edu/orf/) was used to search for upstream open reading frames (uORFs) in the FTO gene.

\section{DNA methylation analysis by pyrosequencing}

Primers were designed using PyroMark Assay Design 2.0 software (Qiagen) and the reverse primers were 5'-labelled with biotin. Details of PCR amplification are given in Table 3. Briefly, $500 \mathrm{ng}$ DNA (isolated from blood) was bisulfite-converted using the EZ DNA Methylation Gold kit (Zymo Research) and amplified with touchdown PCR; in the first eight cycles, the annealing temperature was decreased by $0.5^{\circ} \mathrm{C}$ per cycle, starting at $58^{\circ} \mathrm{C}$, and the next 32 cycles were run at $54^{\circ} \mathrm{C}$. The quality of PCR products was tested by agarose electrophoresis. The methylated control was prepared using CpG methyltransferase (M.SssI, Thermo Scientific) with the following modifications: a threefold increase in M.SssI levels and incubation time at $37^{\circ} \mathrm{C}$. The nonmethylated control was prepared using the REPLI-g Mini Kit (Qiagen). The biotinylated strands of the amplicons were captured on streptavidin-coated beads and incubated with sequencing primers. The specific amplicons were then pyrosequenced on the PyroMark Q24 System (Qiagen), following the manufacturer's protocol.

\section{Statistical analysis}

Association analysis with representative biallelic sites in sample set A was conducted using the Fisher exact test and Plink2 (Chang et al., 2015). We used a false discovery rate (FDR) of $5 \%$ as the cutoff for significance. The association between DNA polymorphism, BCS (BCS 3, 4, 5), and body weight (25-50 kg) in sample set B was studied by a linear regression model adjusted by age (12-180 months) and sex. Association was computed assuming an allelic additive model for 0 , 1, or 2 copies of the allele increasing the trait under study. Prior to the association tests, the effect of neutering status and two-way interactions were examined and excluded from the model as insignificant. The final general statistical model was as follows: 


$$
y=\beta_{0}+\beta_{1} \times s+\beta_{2} \times a+\beta_{3} \times g+e
$$

where:

$y$ is body weight (or BCS),

$s$ is the fixed effect of sex (0 or 1$)$,

$a$ is age (in months),

$g$ is allele content $(0,1$ or 2$)$.

To assess empirical power of association test we conducted a power analysis by randomly permuting genotypes 5000 times. The null hypothesis - that the HardyWeinberg equilibrium holds - was tested using the chi-squared test. Methylation levels in sample set $\mathrm{C}$ were compared with the Mann-Whitney rank sum test. All analysis was carried out in $\mathrm{R}$ using the packages stats, car, and Hardy-Weinberg (R Core Team, 2017).

\section{Results}

\section{Targeted next generation sequencing (tNGS)}

We targeted a region on chromosome 2 (CFA2) harboring functional candidate genes $(F T O$ and $I R X 3)$ in 16 lean $(\mathrm{BCS}=3)$ and 16 obese $(\mathrm{BCS}=5)$ Labradors (sample set A, Figure 2). In total, $3.794 \mathrm{Mb}$ on CFA2 were sequenced (CanFam3.1: 61,545,000-65,339,000). The long fragment upstream of FTO was also sequenced due to a preliminary GWAS experiment (data not shown) tentatively indicating some SNPs, which, however, did not reach genome-wide significance. Later, the GWAS results were reinterpreted and the experiment considered unsuccessful, however, the selected fragment had already been sequenced. Altogether, $84 \%$ of reads passed the trimming process with a 99\% mapping rate. On average, 98\% of the targeted region was covered with at least twelve reads and the mean coverage depth was 255 reads per position. The FTO gene coverage depth was 275 reads ( $>99 \%$ of genes covered by at least twelve reads in each individual), whereas the coverage depth for IRX3 was lower (an average of 54 reads, with $94 \%$ of the gene region covered by at least twelve reads) and varied between dogs $(45 \%-99 \%$ of bases covered by at least twelve reads). The tNGS raw data are available from the European Nucleotide Archive, no. PRJEB20547 (https://www.ebi.ac.uk/ena/data/view/PRJEB20547).

We observed that the nucleotide identity of the canine FTO to the human ortholog is only $56 \%$ (coding sequences $-90 \%$ ), which is a consequence of long introns (approximately $0.4 \mathrm{Mb}$ in both species), whereas the identity between the IRX3 orthologs is $72 \%$ (95\% of coding sequences). In total, we found 12,217 biallelic variants $(10,040$ with a minor allele frequency - MAF > 0.05), including 2051 indels. We detected 1057 intronic variants (1038 with MAF > 0.05) and two 5'UTR variants in FTO, but only two intronic variants (one indel and one SNP) in IRX3. The heterozygosity rate (the relative frequency of heterozygous dogs in the group) tended to vary between the sixteen obese and sixteen healthy dogs. Dogs with BCS $=3$ had lower 
heterozygosity rates (median 0.19) than the dogs with BCS $=5$ (median 0.25). We performed association tests for 272 biallelic variants, but the remaining correlated SNPs $(r=1.0)$ were excluded. After adjusting for multiple testing (FDR), we found no evidence for association between any of the 272 representative variants or BCS status in sample set A (Figure 3).
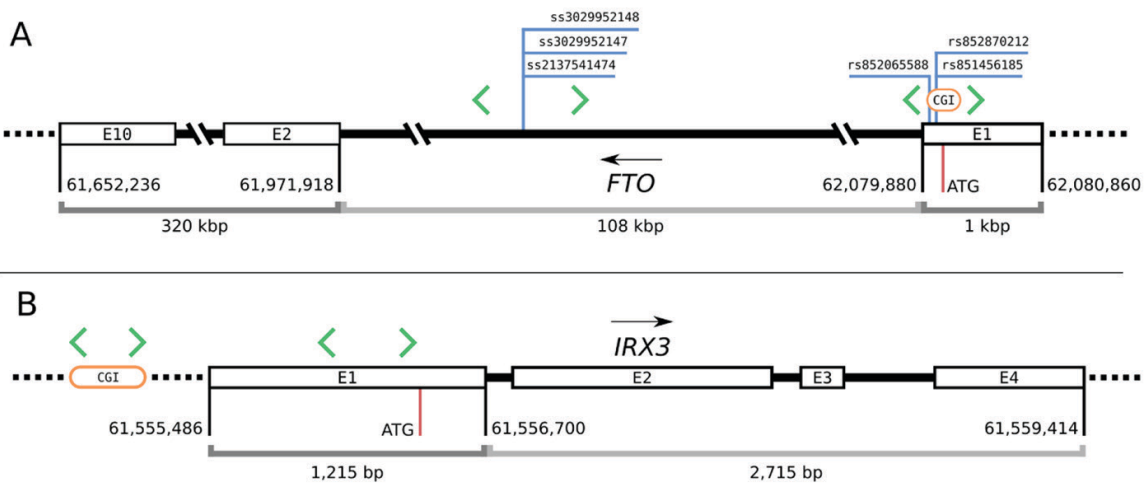

Figure 2. Structure of the two canine candidate genes. Schematic representation of FTO (A) and IRX3 (B) genes; the white boxes represent exons (denoted by a capital letter E followed by the exon number); blue lines indicate the location of the studied variants; red lines mark the start codon location and green angle brackets show the sequenced regions; the examined $\mathrm{CpG}$ islands (CGIs) are indicated by the orange ovals

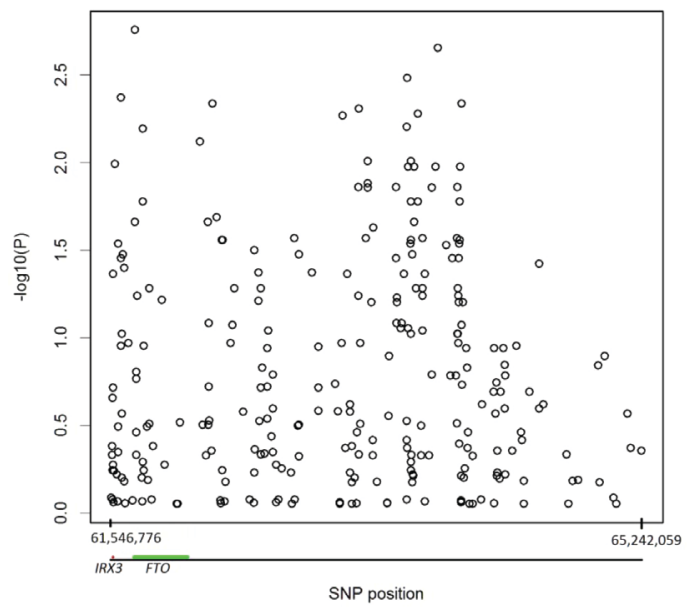

Figure 3. Results of the association study between 272 representative polymorphisms in the sequenced region on canine chromosome 2 and body condition score. Fisher's exact test was applied to the sample of 16 lean and 16 obese Labradors. Having adjusted for multiple testing, no association was found. Uncorrected p-values are shown. Positions of the two candidate genes, FTO (green) and IRX3 (red), are also shown

Candidate gene polymorphism and its association with body weight and BCS

The effect of neutering status and two-way interactions were non-significant and excluded from the statistical model for association study. 
Three candidate regions were selected for the polymorphism search in a larger cohort of Labradors (sample set $\mathrm{B}, \mathrm{N}=165$ ). Two regions were located in FTO: exon 1 and intron 1 (Figure 2). The analyzed region in intron 1 is orthologous to the human sequence harboring a SNP representative for a cluster of polymorphisms associated with obesity (Smemo et al., 2014; Yang et al., 2017). In the IRX3 gene the studied fragment corresponding to a region covering a known obesogenic polymorphism (rs3751723) in humans (Srivastava et al., 2017) overlapped 409bp (365bp of 5'UTR and 44bp of an adjacent coding region) and showed a high (93\%) identity with its human ortholog (Figure 2). Moreover, our in silico studies have shown that the orthologous sequence in 5'UTR of canine $I R X 3$, if polymorphic, would harbor the Myc-Max transcription factor site.

Table 4. Allelic and genotype frequencies at six polymorphic sites in the canine FTO gene

\begin{tabular}{|c|c|c|c|c|c|c|c|c|}
\hline \multirow{2}{*}{$\frac{\text { Polymorphism }}{\text { c. } 23 \mathrm{C}>\mathrm{T}}$} & \multicolumn{3}{|c|}{ Number of animals } & \multicolumn{2}{|c|}{ Allele frequency } & \multicolumn{3}{|c|}{ Genotype frequency } \\
\hline & $\mathrm{CC}$ & $\mathrm{CT}$ & TT & $\mathrm{C}$ & $\mathrm{T}$ & $\mathrm{CC}$ & $\mathrm{CT}$ & TT \\
\hline $\begin{array}{l}\text { rs852870212 } \\
\mathrm{N}=165\end{array}$ & 118 & 40 & 7 & 0.836 & 0.164 & 0.700 & 0.274 & 0.026 \\
\hline c. $26 \mathrm{~A}>\mathrm{G}$ & $\mathrm{AA}$ & $\mathrm{AG}$ & GG & $\mathrm{A}$ & G & AA & $\mathrm{AG}$ & GG \\
\hline $\begin{array}{l}\text { rs851456185 } \\
\mathrm{N}=165\end{array}$ & 164 & 1 & 0 & 0.997 & 0.003 & 0.994 & 0.006 & 0.000 \\
\hline c. $88 \mathrm{C}>\mathrm{T}$ & $\mathrm{CC}$ & CT & TT & $\mathrm{C}$ & $\mathrm{T}$ & $\mathrm{CC}$ & CT & TT \\
\hline $\begin{array}{l}\text { rs852065588 } \\
N=165\end{array}$ & 164 & 1 & 0 & 0.997 & 0.003 & 0.994 & 0.006 & 0.000 \\
\hline c. $220-33577$ delA & $\mathrm{del} / \mathrm{del}$ & del/ins & ins/ins & del & ins & $\mathrm{del} / \mathrm{del}$ & del/ins & ins/ins \\
\hline $\begin{array}{l}\text { ss3029952148 } \\
N=162\end{array}$ & 6 & 6 & 150 & 0.056 & 0.944 & 0.003 & 0.105 & 0.892 \\
\hline c. $220-33549 \mathrm{C}>\mathrm{T}$ & $\mathrm{CC}$ & CT & $\mathrm{TT}$ & $\mathrm{C}$ & $\mathrm{T}$ & $\mathrm{CC}$ & $\mathrm{CT}$ & $\mathrm{TT}$ \\
\hline $\begin{array}{l}\text { ss3029952147 } \\
N=160\end{array}$ & 152 & 7 & 1 & 0.972 & 0.028 & 0.944 & 0.055 & 0.001 \\
\hline c.220- & $\mathrm{del} / \mathrm{del}$ & $\mathrm{del} /$ ins & ins/ins & del & ins & $\mathrm{del} / \mathrm{del}$ & del/ins & ins/ins \\
\hline $\begin{array}{l}33485 \_33484 \text { ins } \mathrm{T} \\
\mathrm{ss} 2137541474 \\
\mathrm{~N}=160\end{array}$ & 154 & 6 & 0 & 0.981 & 0.019 & 0.963 & 0.037 & 0.000 \\
\hline
\end{tabular}

We found six polymorphic sites in FTO: five (rs851456185, rs852065588, ss3029952148, ss3029952147, ss2137541474) with a low MAF (0.003-0.056) and one SNP in the coding sequence (c.23C $>$ T, rs852870212, MAF $=0.16$, Table 4$)$ that was suitable for the association study. No polymorphic site was found in the region orthologous to the human FTO sequence harboring the cluster of SNPs associated with human obesity. We also found no polymorphism in the studied canine IRX3 region. We next analyzed the association of the c.23C $>\mathrm{T}$ (rs852870212) substitution in FTO with body weight and BCS in the sample set $\mathrm{B}(\mathrm{N}=165)$. This polymorphism showed no deviation from Hardy-Weinberg equilibrium. According to the permutation analysis, the observed genotype distribution provided $98.7 \%$ chance to detect an effect of 1.0 phenotypic standard deviation (SD) and 54.0\% chance for 0.5 SD. No significant correlation was found between the c.23C $>\mathrm{T}$ ( $\mathrm{rs} 852870212)$ substitution and BCS or body weight. 


\section{In silico studies}

The C $>$ T polymorphism at position c.88 in FTO (CanFam3.1: 62,079,972; rs852065588) is interesting because, according to the current annotation, the c.88T variant is predicted to introduce a premature TGA stop codon, resulting in a 29-amino-acid (aa) truncated polypeptide, instead of the normal 551-aa polypeptide. As this is unlikely to be true, we carefully examined the sequenced fragment and identified a downstream ATG codon $(62,079,924-2$, c.136_138) that we propose is the correct start codon (Figure 4). The sequence alignment (needle algorithm, EMBOSS) of the predicted protein reveals $89.8 \%$ identity with human FTO for the newly proposed ORF, but only $82.5 \%$ for the currently annotated ORF. This suggests that the position of start ATG codon in the canine FTO reference sequence $(62,080,059-7)$ has been identified incorrectly, and that the downstream ATG codon (at positions: 62,079,9242, c.136_138) is the correct one. Note that, unlike the human FTO annotation, the current annotation for the canine FTO is not based on experimental evidence on the protein level. Further, as a consequence of the new location of the start codon, the upstream ATG codon (the start codon in the current annotation at 62,080,059-7), conditional on the c.88T variant $(62,079,972)$, creates a $90 \mathrm{bp}$ upstream ORF (uORF1, at 62,080,059-62,079,970). Moreover, the c.23T variant (62,080,037; rs852870212), together with the c.88T variant $(62,079,972$; rs852065588), creates another $69 \mathrm{bp}$ uORF (uORF2, at 62,080,038-62,079,970 localization) - Figure 4.

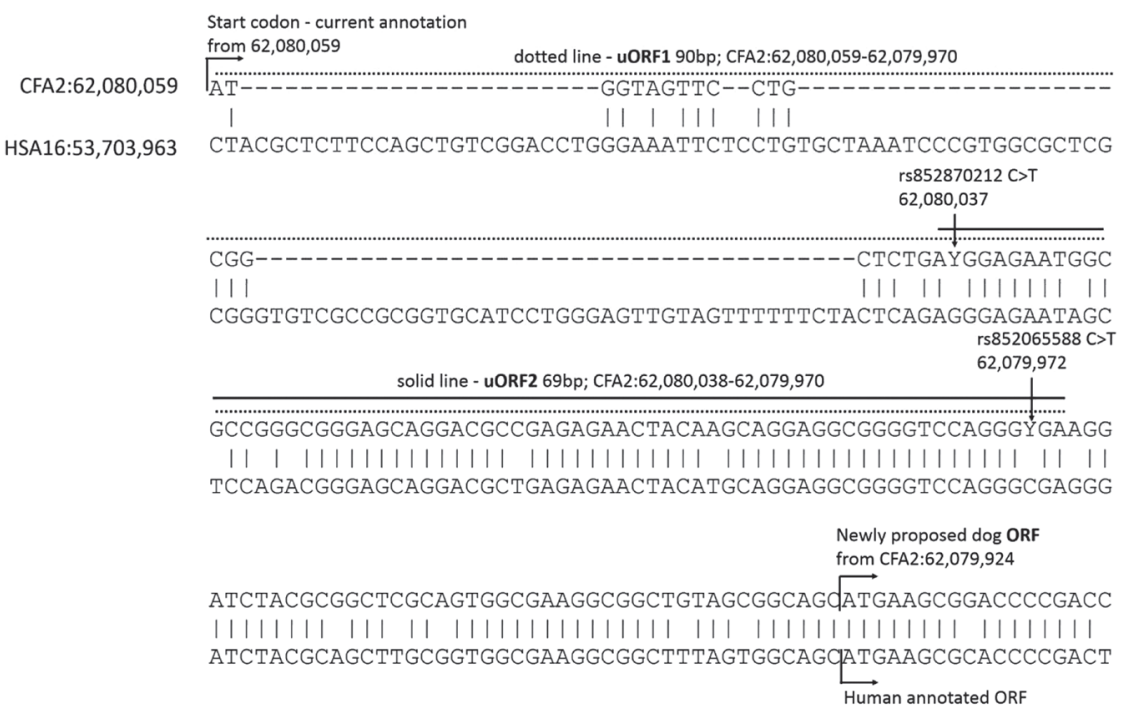

GCAGAGGAAAGAGAGCGCGAAGCTAAG
| CFA2:62,079,880
GCCGAGGAACGAGAGCGCGAAGCTAAG

Figure 4. Alignment of canine and human exon 1 of FTO. The positions of the newly proposed open reading frame (ORF), two upstream ORFs (uORF), and two identified SNPs within the canine FTO gene are shown. All positions are given according to CanFam3.1 and GRCh38.p12 for dog and human assembly, respectively 


\section{DNA CpG methylation analysis}

We selected two CpG islands (CGIs): located in exon 1 of the FTO and in a 5 '-flanking region of the $I R X 3$ gene (Figure 2), for DNA methylation analysis in fifteen lean $(\mathrm{BCS}=3)$ and thirteen obese $(\mathrm{BCS}=5)$ Labradors (sample set $\mathrm{C})$. Seven and six cytosines were analyzed in FTO and IRX3, respectively. In both CGIs, the mean percentage of methylated cytosines was low, ranging from $7 \%$ to $15 \%$ in $F T O$ and from $10 \%$ to $25 \%$ in $I R X 3$ (Figure 5). Interestingly, the methylation of the seven FTO cytosines was highly correlated, while the methylation of the six IRX3 cytosines was not. We did not detect any significant difference in $\mathrm{CpG}$ methylation between the lean and obese groups. Moreover, our data suggests the methylation level in FTO and IRX3 has no relation to age, sex, or neutering.

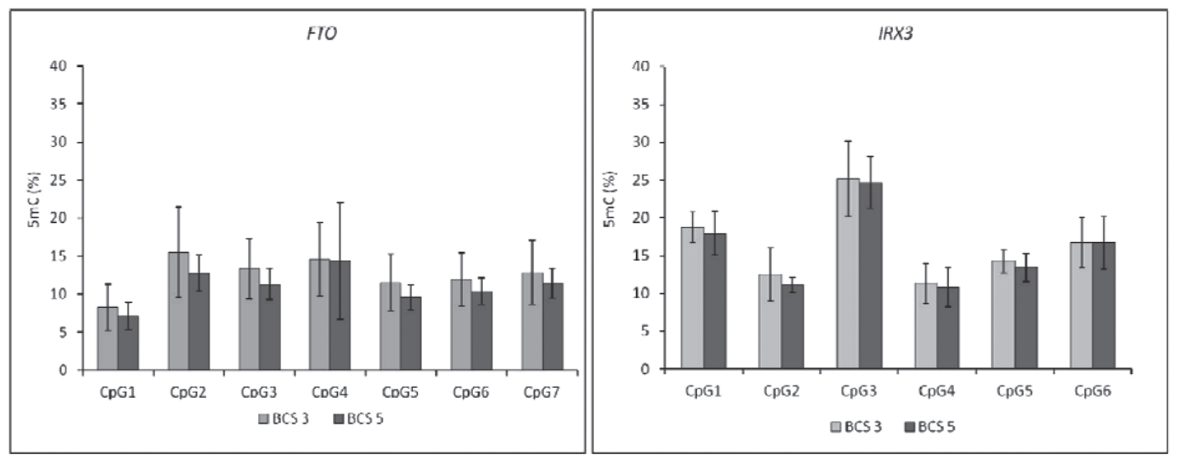

Figure 5. Results of the association study on methylation level in two candidate genes (FTO and IRX3) and obesity. Methylation was studied in 15 lean $(\mathrm{BCS}=3)$ and 13 obese $(\mathrm{BCS}=5)$ Labrador dogs. No statistically significant differences were found between the lean and obese dogs. The mean percentage of methylation $\pm \mathrm{SD}$ in the examined $\mathrm{CpG}$ islands is shown. The particular cytosines in each analyzed fragment are indicated by $\mathrm{CpG} 1, \mathrm{CpG} 2$, etc.

\section{Discussion}

Studies on human obesity over the past decade have identified more than 220 genetic markers (Pigeyre et al., 2016; Singh et al., 2017), however, a genetic contribution to canine obesity is poorly recognized (Switonski and Mankowska, 2013; Yeo, 2017). Limited studies on this disease have indicated the presence of breedspecific gene pools responsible for obesity in dogs. As human FTO, MC4R, POMC, $B D N F$, and PCSK1 genes show the most pronounced effects (Pigeyre et al., 2016), these genes have been considered as candidates for canine obesity. Recently, a strong effect predisposing to obesity of Labrador retrievers and the closely related Flatcoated retrievers was reported for a 14 bp deletion in POMC (Raffan et al., 2016; Mankowska et al., 2017 a). Another candidate, the $M C 4 R$ gene, showed no association with obesity (Mankowska et al., 2017 b) or with morphological measures in Golden retrievers (van den Berg et al., 2010), but is tentatively associated with 
body weight in Beagles (Zeng et al., 2014). These findings are expected because of selective dog breeding which means that within breeds, genetic determinants of even complex disease can be very different to those in other breeds. Therefore we limited our association study to a single obesity-prone breed. On the contrary, Miyabe et al. (2015) analyzed a multibreed cohort and detected a significant effect of canine GPR120 gene on BCS.

Based on our sample of Labrador retriever dogs we collected a suggestive evidence that human and canine FTO genes may have different contribution to obesity. The widely studied FTO polymorphisms located in intron 1 show associations with body weight, BMI, and body fat distribution in multiple human populations (Hardy et al., 2010; González et al., 2012; Zermeño-Rivera et al., 2014). In our study, we resequenced the orthologous region in intron 1 of the canine FTO gene, but found no polymorphism. Alternatively, this result may suggest that the largest determinant of polygenic obesity in humans is not having a detectable effect in a single dog breed.

Although the well-known effect of human FTO was not found in our sample of dogs, we discovered that two SNPs in the canine FTO create two different uORFs. It is known that UORFs are translated in the same manner as major ORFs into a short polypeptide that may interfere with the subsequent translation of the major ORF, due to its inability to reinitiate ribosome subunits after uORF translation (Somers et al., 2013). As a result, the expression of the major ORF can be reduced by as much as $80 \%$ (Calvo et al., 2009). Interestingly, no association was observed with BCS or body weight of the c. $23 \mathrm{C}>\mathrm{T}$ polymorphism (rs852870212) creating the uORF in the canine FTO gene. It should be pointed out that the minor variant (c.23T) occurred in the studied cohort with a low frequency $(0.16)$, and thus our study had a limited power to find similar effect of FTO to that seen in human populations. However, we gave an evidence, by applying permutation power analysis, that the studied sample set had a reasonable size to look for a larger allelic effect.

Smemo et al. (2014) showed that FTO and IRX3 genes interact with each other. A multianalytical approach of 55 SNPs in 28 obesity-related human genes has confirmed the strong linkage disequilibrium (LD) between SNPs in FTO and IRX3, along with polymorphisms in TCF7L2 and TMEM18 genes, indicating that they are the major SNPs contributing to BMI in the North Indian population (Srivastava et al., 2017). Based on these results and the fact that the G>T SNP (rs3751723) located in the 5'UTR of human IRX3 is highly associated with the BMI index, we decided to study the corresponding region of 5'UTR in obese dogs. However, in spite of the high sequence similarity between the orthologous regions in the human and canine genomes (256 bp with $94.53 \%$ identity), no such polymorphism was found in Labrador dogs in this study. The association between human obesity and IRX3 polymorphisms has been reported for a Chinese population. It has been observed that the rs8053360 (C>T) SNP is associated with birth weight in females (resulting in an increase with CC genotype) (Liu et al., 2018). Additionally, GG genotype for rs1126960 increased birth weight in females.

Several studies have reported correlations between the methylation pattern in blood cells and obesity (Campión et al., 2009; Crujeiras et al., 2013, 2017). Also a recent report has shown a difference in $\mathrm{CpG}$ methylation in PTPRS and PER3 
genes between obese and nonobese children (Samblas et al., 2018). We observed very low cytosine methylation levels in both analyzed CGIs. The CGI studied in the FTO gene was localized in the structural regions (exon 1). The role of DNA methylation within the gene body is still unclear, but there is evidence that it influences transcriptional elongation and alternative splicing (Lee et al., 2015).

The well-known contributors to canine obesity include age, sex and gonadal status (neutered or intact) (German, 2006). Our data confirmed a significant effect of age and sex, whereas the effect of gonadal status appeared to be non-significant. It should be pointed out that study of Lefebvre et al. (2013) carried out on a larger dog cohort showed that risk for becoming overweight was significantly greater in neutered than in intact dogs, but only during the first 2 years after gonadectomy. In our study the age of dogs ranged from 12 to 180 months and therefore only a part of the sample was at increased risk of being overweight due to neutering.

\section{Conclusions}

Our study suggests that, unlike in humans, FTO and IRX3 are not promising markers of obesity in Labradors. However, the two identified upstream reading frames (uORFs) created by two SNPs deserve further study in a larger cohort of obese and lean Labradors. Based on in silico study we propose an improvement in the current annotation of FTO. Additionally, DNA methylation of short CpG islands in FTO and IRX3 showed no association with obesity.

\section{Acknowledgments}

We cordially thank Agata Biedna and Aneta Dorawa for their laboratory work.

\section{References}

Berg L. van den, van den Berg S.M., Martens E.E.C.P., Hazewinkel H.A.W., Di jkshoorn N.A., Delemarre-van de Waal H.A., Heutink P., Leegwater P.A.J., Heuven H.C.M. (2010). Analysis of variation in the melanocortin-4 receptor gene (mc4r) in golden retriever dogs. Anim. Genet., 41: 557.

B olger A.M., L o h s e M., Us a d e 1 B. (2014). Trimmomatic: A flexible trimmer for Illumina sequence data. Bioinformatics, 30: 2114-2120.

Calvo S.E., Pagli ar in i D.J., M o o th a V.K. (2009). Upstream open reading frames cause widespread reduction of protein expression and are polymorphic among humans. Proc. Natl. Acad. Sci., 106: 7507-7512.

Campión J., Mila gro F.I., G o y e ne chea E., Martín ez J.A. (2009). TNF- $\alpha$ promoter methylation as a predictive biomarker for weight-loss response. Obesity, 17: 1293-1297.

Chang C.C., Chow C.C., Telli er L.C.A.M., Vat tikuti S., Purcell S.M., L e e J.J. (2015). Second-generation PLINK: Rising to the challenge of larger and richer datasets. Gigascience, 4: 7.

Crujeiras A.B., Campion J., Díaz-Lagares A., Milagro F.I., Goyenechea E., A b e te I., C a s a nu e va F.F., M artín e z J.A. (2013). Association of weight regain with specific methylation levels in the NPY and POMC promoters in leukocytes of obese men: A translational study. Regul. Pept., 186: 1-6.

Crujeiras A.B., Diaz-Lagares A., Sandoval J., Milagro F.I., Navas-Carretero S., Carreira M.C., Gomez A., Hervas D., Monteiro M.P., Cas anueva F.F., Esteller M., Martinez J.A. (2017). DNA methylation map in circulating leukocytes mir- 
rors subcutaneous adipose tissue methylation pattern: A genome-wide analysis from non-obese and obese patients. Sci. Rep., 7: 41903.

Frayling T.M., Timps on N.J., We edon M.N., Freathy R.M., Lindgren C.M., Perry J.R.B., Katherine S., Lango H., Rayner N.W., Shields B., Harries L.W., Barrett C., Ellard S., Groves C.J., Knight B., Patch A., Nes s A.R., Ebrahim S., Law lor D.A., Ring S.M., B en-shlomo Y., Jarvelin M., Sovio U., B ennett A.J., Melzer D., L oos R.J.F., B arroso I., Wareham N.J., Karpe F., Ow en K.R., Cardon L.R., Walker M., Hitman G.A., Colin N.A., Doney A.S.F., Morris A.D., Smith G.D., Wellcome T., Case T., Consortium C., Hattersley A.T., Mcc a r th y M.I. (2007). A common variant in the FTO gene is associated with body mass index and predisposes to childhood and adult obesity. Science, 316: 889-894.

G e r m a n A.J. (2006). The growing problem of obesity in dogs and cats. J. Nutr., 136: 1940S-1946S.

G e r m a n A.J. (2016). Weight management in obese pets: The tailoring concept and how it can improve results. Acta Vet. Scand., 58: 57.

González J.R., González-Carpio M., Hernández-Sáez R., Serrano Vargas V., Hidalgo G.T., Rubio-Rodrigo M., García-Nogales A., Ñez Estévez M., Lu e ng o Pérez L.M., R odríguez - Ló pez R. (2012). FTO risk haplotype among early onset and severe obesity cases in a population of Western Spain. Obesity, 20: 909-915.

G o s s e 11 in J., Wre n J.A., S un de r l a n d S.J. (2007). Canine obesity - an overview. J. Vet. Pharmacol. Ther., 30: 1-10.

Hardy R., Wills A.K., Wong A., Elks C.E., Wareham N.J., Loos R.J.F., Kuh D., O n g K.K. (2010). Life course variations in the associations between FTO and MC4R gene variants and body size. Hum. Mol. Genet., 19: 545-552.

Huang R.C., Garratt E.S., P an H., Wu Y., Davis E.A., B arton S.J., Burdge G.C., God frey K.M., Holbrook J.D., Lillycrop K.A. (2015). Genome-wide methylation analysis identifies differentially methylated $\mathrm{CpG}$ loci associated with severe obesity in childhood. Epigenetics, 10: 995-1005.

L e e S.-M., Cho i W.Y., L e e J., K i m Y.-J. (2015). The regulatory mechanisms of intragenic DNA methylation. Epigenomics, 7: 527-531.

L e fe bvre S.L., Yang M., Wang M., E 11 i o t t D.A., B u ff P.R., L und E.M. (2013). Effect of age at gonadectomy on the probability of dogs becoming overweight. J. Am. Vet. Med. Assoc., 243: 236-243.

L i H., D u r b in R. (2010). Fast and accurate long-read alignment with Burrows-Wheeler transform. Bioinformatics, 26: 589-595.

Liu C., Chu C., Zhang J., Wu D., Xu D., Li P., Chen Y., Li u B., P e i L., Zhang L., Liu S., Q i T., L o u X.Y., Li L. (2018). IRX3 is a genetic modifier for birth weight, adolescent obesity and transaminase metabolism. Pediatr. Obes., 13: 141-148.

L o o s R.J.F., Y e o G.S.H. (2014). The bigger picture of FTO - The first GWAS-identified obesity gene. Nat. Rev. Endocrinol., 10: 51-61.

Mank ow ska M., St a chowiak M., Graczyk A., Ciazynska P., Gogulski M., Nizan s ki W., Swit on ski M. (2016). Sequence analysis of three canine adipokine genes revealed an association between TNF polymorphisms and obesity in Labrador dogs. Anim. Genet., 47: 245-249.

Mank ow ska M., Krze minska P., Gra c zy k M., Sw it on s ki M. (2017 a). Confirmation that a deletion in the POMC gene is associated with body weight of Labrador Retriever dogs. Res. Vet. Sci., 112: 116-118.

Mankowska M., Nowacka-Woszuk J., Graczyk A., Ciazynska P., Stachow i a k M., S w it o n s k i M. (2017 b). Polymorphism and methylation of the $M C 4 R$ gene in obese and non-obese dogs. Mol. Biol. Rep., 44: 333-339.

Mi y abe M., G in A., On ozaw a E., Da imon M., Yamada H., Oda H., Mori A., Momota Y., Azakami D., Yamamoto I., Mochizuku M., Sako T., Tamura K., Ishio$\mathrm{k}$ a K. (2015). Genetic variants of the unsaturated fatty acid receptor GPR120 relating to obesity in dogs. J. Vet. Med. Sci., 77: 1201-1206.

O s t o M., L u t z T.A. (2015). Translational value of animal models of obesity - Focus on dogs and cats. Eur. J. Pharmacol., 759: 240-252.

Pigeyre M., Yazdi F.T., Ka ur Y., Meyre D. (2016). Recent progress in genetics, epigenetics and metagenomics unveils the pathophysiology of human obesity. Clin. Sci., 130: 943-986. 
Raffan E., Denn is R.J., O'D on ov an C.J., B e c ker J.M., S c o t t R.A., S m ith S.P., With ers D.J., Wo od C.J., Conci E., Cle ments D.N., S u m mers K.M., German A.J., M e 1 lersh C.S., Arendt M.L., Iyemere V.P., Withers E., Söder J., Werners on S., Andersson G., Lindblad-Toh K., Yeo G.S.H., O'Rahilly S. (2016). A deletion in the canine $P O M C$ gene is associated with weight and appetite in obesity-prone Labrador Retriever dogs. Cell Metab., 23: 893-900.

S a mblas M., Milagro F.I., Manse go M.L., Marti A., Martinez J.A. (2018). PTPRS and PER3 methylation levels are associated with childhood obesity: Results from a genome-wide methylation analysis. Pediatr. Obes., 13: 149-158.

S ing h R.K., K u m a r P., M a h a 1 ing a m K. (2017). Molecular genetics of human obesity: A comprehensive review. C. R. Biol., 340: 87-108.

S m e mo S., Ten a J.J., K i m K.H., Gamazon E.R., S a kabe N.J., Gómez-M arín C., A ne a s I., Credidio F.L., S obreir a D.R., Wa s e r m a n N.F., L e e J.H., Puvi ind ran V., Tam D., Shen M., Son J.E., Vakili N.A., Sung H.K., Naranjo S., Acemel R.D., Manzanares M., Nagy A., Cox N.J., Hui C.C., Gomez-Skarmeta J.L., Nóbre g a M.A. (2014). Obesity-associated variants within FTO form long-range functional connections with IRX3. Nature, 507: 371-375.

S o mer s J., Pöyry T., Willi s A.E. (2013). A perspective on mammalian upstream open reading frame function. Int. J. Biochem. Cell Biol., 45: 1690-1700.

Srivastava A., Mitta 1 B., Prakash J., Srivastava P., Srivastava N., Srivastava N. (2017). A multianalytical approach to evaluate the association of 55 SNPs in 28 genes with obesity risk in North Indian adults. Am. J. Hum. Biol., 29.

St a chowiak M., S zczerbal I., Sw itonski M. (2016). Genetics of adiposity in large animal models for human obesity-studies on pigs and dogs. Prog. Mol. Biol. Transl. Sci., 140: 233-270.

S t e e n b e e k F.G. van, H y tön e n M.K., L e e g w a t e r P.A.J., L o h i H. (2016). The canine era: the rise of a biomedical model. Anim. Genet., 47: 519-527.

S w it on s k i M. (2014). Dog as a model in studies on human hereditary diseases and their gene therapy. Reprod. Biol., 14: 44-50.

Switonski M., Mankowska M. (2013). Dog obesity - The need for identifying predisposing genetic markers. Res. Vet. Sci., 95: 831-836.

Van der Auwera G.A., Carneiro M.O., Hartl C., Poplin R., del Angel G., Levy- Moonshine A., Jordan T., Shakir K., Roazen D., Thibault J., Banks E., Gari mella K. V., A lts huler D., Gabriel S., De Pris to M.A. (2013). From fastQ data to highconfidence variant calls: The genome analysis toolkit best practices pipeline. Curr. Protoc. Bioinforma., 43: 11.10.1-11.10.33.

Yang Q., Xi a o T., Gu o J., S u Z. (2017). Complex relationship between obesity and the fat mass and obesity locus. Int. J. Biol. Sci., 13: 615-629.

Ye o G.S.H. (2017). Genetics of obesity: can an old dog teach us new tricks? Diabetologia, 60: 778-783.

Z eng R., Z hang Y., Du P. (2014). SNPs of melanocortin 4 receptor (MC4R) associated with body weight in Beagle dogs. Exp. Anim., 63: 73-8.

Zermeño-Rivera J.J., A s tocondor-Pérez J.P., Valle Y., Padilla-Gutiérrez J.R., Orozco-Castellanos R., Figuera L.E., Gutiérrez-A mavizca B.E. (2014). Association of the FTO gene SNP rs17817449 with body fat distribution in Mexican women. Genet. Mol. Res., 13: 8561-8567.

Z hang Z., S c hw a rtz S., Wa g n e r L., Mi 11 e r W. (2000). A greedy algorithm for aligning DNA sequences. J. Comput. Biol., 7: 203-214.

Received: 22 XI 2018

Accepted: 17 I 2019 